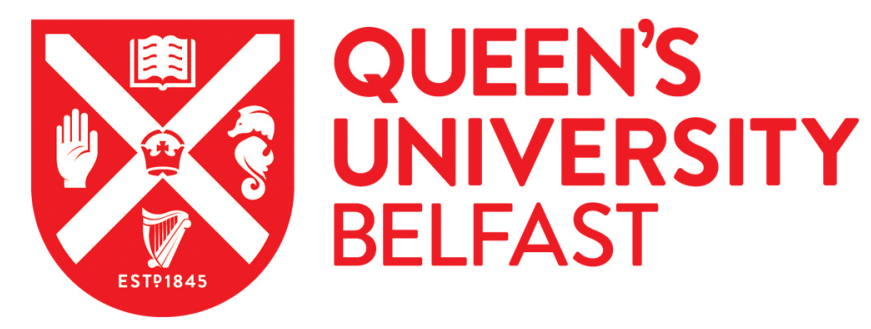

\title{
Domain annihilation due to temperature and thickness gradients in single-crystal BaTiO3
}

McGilly, L., Burnett, T. L., Schilling, A., Cain, M. G., \& Gregg, M. (2012). Domain annihilation due to temperature and thickness gradients in single-crystal BaTiO3. Physical Review B (Condensed Matter), 85(5), 1-5. [054113]. https://doi.org/10.1103/PhysRevB.85.054113

Published in:

Physical Review B (Condensed Matter)

Document Version:

Publisher's PDF, also known as Version of record

Queen's University Belfast - Research Portal:

Link to publication record in Queen's University Belfast Research Portal

Publisher rights

(O2012 American Physical Society. This work is made available online in accordance with the publisher's policies. Please refer to any applicable terms of use of the publisher.

\section{General rights}

Copyright for the publications made accessible via the Queen's University Belfast Research Portal is retained by the author(s) and / or other copyright owners and it is a condition of accessing these publications that users recognise and abide by the legal requirements associated with these rights.

Take down policy

The Research Portal is Queen's institutional repository that provides access to Queen's research output. Every effort has been made to ensure that content in the Research Portal does not infringe any person's rights, or applicable UK laws. If you discover content in the Research Portal that you believe breaches copyright or violates any law, please contact openaccess@qub.ac.uk. 


\title{
Domain annihilation due to temperature and thickness gradients in single-crystal $\mathrm{BaTiO}_{3}$
}

\author{
L. J. McGilly, ${ }^{1, *}$ T. L. Burnett, ${ }^{2}$ A. Schilling, ${ }^{1}$ M. G. Cain, ${ }^{2}$ and J. M. Gregg ${ }^{1}$ \\ ${ }^{1}$ Centre for Nanostructured Media, School of Maths and Physics, Queen's University Belfast, Belfast, BT7 INN, United Kingdom \\ ${ }^{2}$ National Physical Laboratory, Hampton Road, Teddington, TW11 OLW, United Kingdom
}

(Received 1 December 2011; published 24 February 2012)

\begin{abstract}
The manner in which $90^{\circ}$ ferroelectric-ferroelastic domains respond to changes in temperature has been mapped in $\mathrm{BaTiO}_{3}$ single crystals using atomic force microscopy. Domain periodicity remains unaltered until approximately $2{ }^{\circ} \mathrm{C}$ below the Curie temperature $\left(T_{C}\right)$, whereupon domains coarsened dramatically. This behavior was successfully rationalized by considering the temperature dependence of the parameters associated with standard models of ferroelastic domain formation. However, while successful in describing the expected radical increase in equilibrium period with temperature, the model did not predict the unusual mechanism by which domain coarsening occurred; this was not continuous at a local level but instead involved discrete domain annihilation events. Subsequent insights from a combination of free energy analysis for the system and further experimental data from an analogous situation, in which domain period increases with increasing crystal thickness, suggested that domain annihilation is inevitable whenever a component of the relevant gradient that affects domain period is orientated parallel to the domain walls. Consistent with this thesis, we note that, for the observations presented herein, the thermal gradient possessed a significant component parallel to the domain walls. We suggest that domain annihilation is a general feature of domain structures in gradient fields.
\end{abstract}

DOI: 10.1103/PhysRevB.85.054113

PACS number(s): 77.80.Dj, 77.80.B-, 62.20.D-

\section{INTRODUCTION}

The importance of domains and domain-wall motion, in contributing to the technologically useful properties of ferroelectrics cannot be overstated. In ferroelectric random access memory (FRAM), for example, it is the domain orientation within each small-scale capacitor that determines the binary state (" 1 " or " 0 ") for that element in the memory chip. ${ }^{1}$ Equally, rewriting ferroelectric bits requires nucleation of reverse domains and switching through lateral motion of $180^{\circ}$ domain walls. Piezoelectric devices too, often rely on the existence of domains and domain wall movement: extrinsic piezoelectric distortions, which result from the motion of $90^{\circ}$ domain walls, can generate overall strains that are significantly larger than those associated with intrinsic piezoelectric tensors. ${ }^{2}$

Research on ferroelectric domains is not only of technological relevance. It is also of fundamental significance, as there are many aspects of behavior that are not yet fully understood, or are only starting to be revealed: for example, while closure domains form readily, in response to demagnetizing fields, in patterned soft ferromagnetic materials, ${ }^{3-6}$ similar closure states due to depolarizing fields in ferroelectrics are seldom observed. ${ }^{7-14}$ When they do occur, they are either nanoscale in their extent, ${ }^{7,8,10-13}$ or require complex "superdomain-subdomain" hierarchies to offset disclination stresses. ${ }^{14}$ Additionally, while the continuous spatial rotation of dipole orientation, needed for true vortex states to form, has been predicted by atomistic simulation for around a decade, ${ }^{15,16}$ this phenomenon was not directly observed until very recently (using aberration-corrected transmission electron microscopy ${ }^{11,12}$ ).

This article concerns the changes that occur in ferroelectric domains as a function of temperature. Given the strong temperature dependence of many of the other properties of ferroelectrics that display first-order phase transitions, ${ }^{17-19}$ it is perhaps somewhat surprising that domain periodicity is thought to be strongly temperature insensitive; recent LandauGinzburg-Devonshire modeling of $180^{\circ}$ domains in uniaxial ferroelectrics clearly illustrates this point. ${ }^{20,21}$ Experimentally, too, this notion is often borne out by direct observation. ${ }^{22}$ For example, Kalinin and Bonnell found the period of $90^{\circ}$ domains in $\mathrm{BaTiO}_{3}$ to be invariant right up to the point where domain contrast simply disappeared as a result of heating through the Curie temperature $\left(T_{C}\right){ }^{23,24}$ However, studies have shown domains across second order phase transitions in ferroelectrics ${ }^{25}$ and relaxors ${ }^{26-29}$ to be strongly influenced by temperature. Other previous work has researched various aspects of domain behavior as a function of temperature in $\mathrm{BaTiO}_{3}{ }^{30-32}$ and $\mathrm{PbTiO}_{3} .{ }^{33}$

The work presented in this article is largely consistent with the research discussed above, but with some important differences: atomic force microscopy has been used to map the behavior of $90^{\circ}$ stripe domains in bulk tetragonal $\mathrm{BaTiO}_{3}$ as a function of temperature with more modern, temperature-stable apparatus than was widely available previously. On heating, no change in domain configuration or periodicity was found until within $\sim 2{ }^{\circ} \mathrm{C}$ of $T_{C}$. In a relatively small temperature window, we observe that the domain periodicity increases dramatically. We have rationalized this behavior successfully by considering established models for ferroelastic domains, but incorporating the temperature-dependence of model variables. We also note that changes in domain periodicity are accommodated through domain annihilation: a feature that we attribute to the orientation of the thermal gradient with respect to the $90^{\circ}$ domain walls. Comparison between the observations made under this thermal gradient and those made in thin $\mathrm{BaTiO}_{3}$ platelets under a thickness gradient, demonstrate that changing domain period through domain annihilation is a general feature when gradient vectors are not perpendicular to the domain walls with which they interact.

\section{EXPERIMENTAL METHODOLOGY}

A commercially sourced $\mathrm{BaTiO}_{3}$ single crystal with $\{100\}_{\text {pseudocubic(pc) }}$ bounding surfaces and approximate 
dimensions $5 \times 5 \times 0.5 \mathrm{~mm}^{3}$, was investigated using a Bruker Icon atomic force microscope (AFM) incorporating a Digital Instruments Thermal Application Controller (TAC) with associated thermoelectric heating stage. The TAC allowed for precise, stable control of heating stage temperature setpoint with a precision of $\sim 0.1{ }^{\circ} \mathrm{C}$. The temperatures reported in this paper were measured using a thermocouple attached to the underside of the sample. The temperature of the crystal surface, pertinent to the images recorded, is estimated to be approximately $10{ }^{\circ} \mathrm{C}$ lower than the setpoint of the TAC. AFM probes with a force constant of $\sim 3 \mathrm{Nm}^{-1}$ (Bruker SCM-PIT) were used for all experiments. In order to preserve probe tip quality, the single crystal was annealed at $200{ }^{\circ} \mathrm{C}$ for 30 minutes immediately prior to the experiment in an attempt to remove volatile contaminants from the sample surface. Optical microscopy, which gave information over a large field of view, was employed simultaneously with the detailed and quantitative nanoscale measurements from AFM. In this manner, precise temperature control of the single-crystal sample allowed experimental investigation of domain states within $2{ }^{\circ} \mathrm{C}$ of the ferroelectric-to-paraelectric phase transition.

In addition, so that the effect of thickness variations on domain period could be investigated by scanning transmission electron microscopy (STEM), thin lamellar sheets of $\mathrm{BaTiO}_{3}$ with thickness gradients were produced by focused ion beam (FIB) milling as described previously. ${ }^{34}$ These samples were imaged using a high-angle annular dark field (HAADF) detector on a FEI Tecnai F20 microscope.

\section{RESULTS AND DISCUSSION}

A set of $90^{\circ}$ "a-c" stripe domains, with $\{101\}_{\mathrm{pc}}$ walls that intersect the $\{100\}_{\mathrm{pc}} \mathrm{BaTiO}_{3}$ surface along $\langle 100\rangle_{\mathrm{pc}}$ directions, can be seen in Fig. 1(a). The surface deformation usually

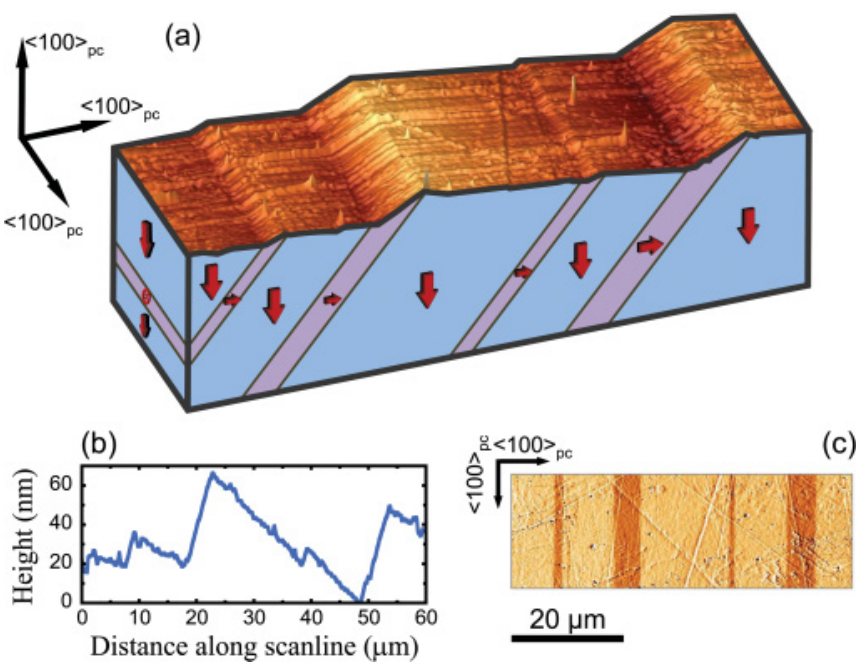

FIG. 1. (Color online) Surface corrugation due to the a-c domain structure is shown in the $3 \mathrm{D}$ representation of AFM topography combined with a schematic cutaway from within the depth of the sample (a). A scanline profile from (a) shows the height of the surface deformation (b). The image in (c) is the associated deflection/error signal from (a).

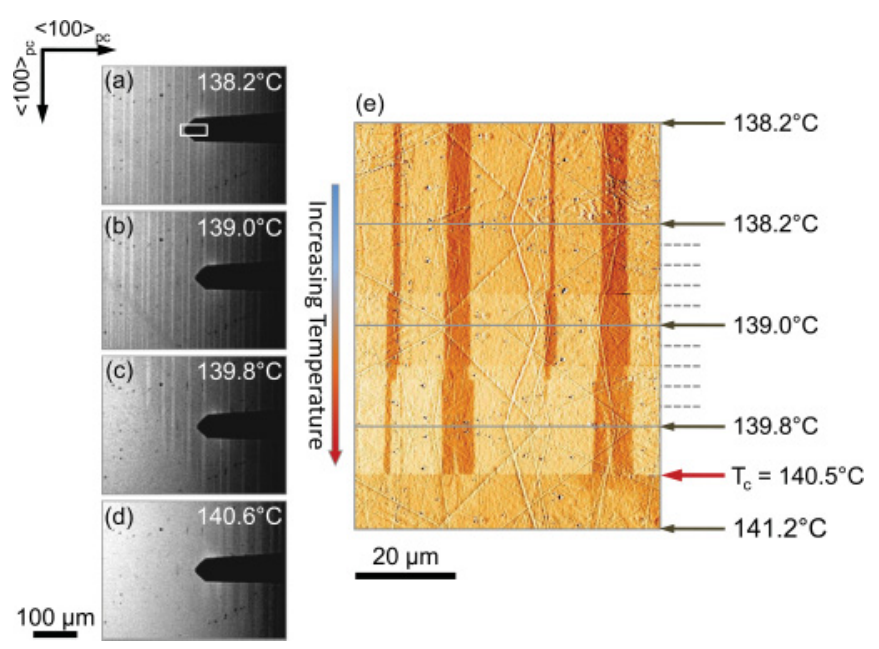

FIG. 2. (Color online) Optical microscope images (a)-(d) show the a-c domain structure at increasing temperatures. AFM deflection images (e), scanned approximately within the white boxed region in (a), showing fine structure evolution with increasing temperature near the phase transition at a nominal temperature $T_{c}=140.5^{\circ} \mathrm{C}$ (temperatures recorded were those at the underside of the $\mathrm{BaTiO}_{3}$ single crystal). The grey dotted lines on the right show approximate $0.2^{\circ} \mathrm{C}$ step increases of temperature. N.B. This composite is composed of four images continuously captured one after another and arranged such that the sense of increasing temperature is retained.

associated with this type of domain is clearly evident. The "corrugation" that is displayed in the line profile of Fig. 1(b) arises from the tetragonality of the unit cell and has been treated in detail elsewhere. ${ }^{23,35}$ The corresponding deflection error image (which records the derivative of the topographic " $z$ deflection" with respect to cantilever displacement) in Fig. 1(c), shows the domains clearly; this mode of imaging, and the data extracted from it, was used to monitor domain behavior throughout the AFM work presented.

The optical micrographs [see Figs. 2(a)-2(d)], taken at $0.8^{\circ} \mathrm{C}$ intervals of increasing temperature, show the domains and their evolution near the phase transition. The domain images presented in Figs. 2(a) and 2(b) show that there is no appreciable change between set temperatures on the TAC of $138.0^{\circ} \mathrm{C}$ and $138.8^{\circ} \mathrm{C}$ (also true for temperatures below $138.0^{\circ} \mathrm{C}$, not shown). Figure 2(c), however, shows the onset of the paraelectric phase (absence of domain structure on the lower left-hand side of the image) and an apparent approximate doubling of the domain period close to the AFM tip. Evidently, from this figure, there is a thermal gradient that should be at a high angle to the isothermal contour, indicated by the junction between the region in which domains can be seen and that where they are absent. The effects of this thermal gradient on domain behavior form a major part of the discussion of this paper. Progressive migration of the paraelectric-ferroelectric interface on increasing temperature can be seen in Fig. 2(d). At $140.5^{\circ} \mathrm{C}$ [see Fig. 2(e)], there is no longer any domain contrast within the AFM scanning region. The optical micrographs show that domains may still be present under the AFM cantilever but even these vanish by $141^{\circ} \mathrm{C}$. No changes in the AFM images were observed above $140.5^{\circ} \mathrm{C}$. 
(a)

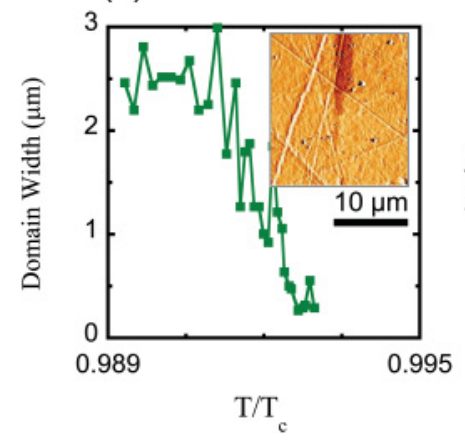

(b)

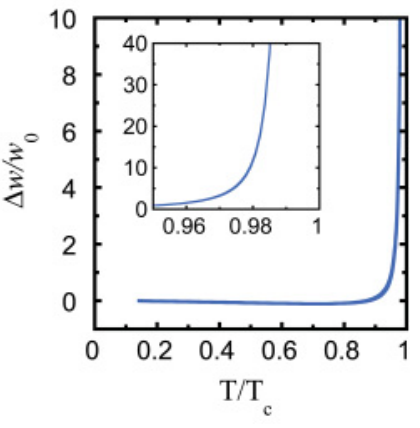

FIG. 3. (Color online) Domain width, for the domain that undergoes an annihilation event, is plotted as a function of temperature in (a) consistent with the inset image. In (b) the fractional change in domain width over that at room temperature $\left(\Delta w / w_{0}\right)$ as a function of temperature (expressed as a fraction of $T_{c}$ ) is shown, as calculated from established models but incorporating temperature-dependent parameters. The inset shows the calculated dependency close to $T_{c}$.

Insight into the abrupt change in domain period just below $\mathrm{T}_{\mathrm{C}}$ can be gained from analysis of the AFM deflection images shown in Fig. 2(e). In this figure the temperature was increased in steps during scanning so that "real-time" data acquisition reveals information on the response of the domains with high resolution, thermally, temporally, and spatially. Beginning at approximately $139.4{ }^{\circ} \mathrm{C}$, and then developing over a temperature range less than $0.4^{\circ} \mathrm{C}$, the disappearance or "annihilation" of an "a" domain was observed [see Figs. 2(e) and 3(b)]. Such annihilation events, where pairs of $90^{\circ}$ domain walls coalesce, facilitate the dramatic increase in the domain period seen using optical microscopy [see Figs. 2(a) to 2(c)]. Figure 3(a) plots the width of the thin "a" domain as a function of temperature. It is apparent that for this domain, domain wall coalescence involves the movement of only one wall. Whilst it is not resolved even in these quite rapidly scanned images (time per line $\sim 1 \mathrm{sec}$ ) we would expect the domain annihilation event to be mediated by a wedge-shaped domain front similar to that proposed in work by Salje and Ishibashi ${ }^{36}$ and partially captured in Fig. 2(e). Additionally, work by Takashige et al. has noted similar discontinuities in domain period with temperature. ${ }^{37}$

In an attempt to understand the reason for changes in domain period with increasing temperature, a semiempirical model was considered, based on previous theoretical treatments of ferroelastic $90^{\circ}$ domains in thin films..$^{34,38-41}$ Even though the $\mathrm{BaTiO}_{3}$ imaged in the current work was a bulk single crystal, the expressions developed for thin films become increasingly relevant as film thickness tends to infinity. In this case, the terms in the originally developed expressions associated with the mismatch stress between film and substrate are replaced by the spontaneous strain in the ferroelastic itself. ${ }^{42}$ The model expression considered was therefore

$$
w(T)=k\left[\frac{\gamma(T) d}{E(T) s(T)^{2}}\right]^{1 / 2},
$$

where $w(T)$ is the temperature-dependent domain width, $k$ is a constant, $d$ is the crystal thickness, $\gamma(T), E(T)$, and $s(T)$ are the temperature-dependent values of, respectively, domain wall energy density (taken from calculated values of Marton et al..$^{43}$ ), Young's modulus (values derived from Dong et $a l .{ }^{44}$ ), and the spontaneous strain (data taken from Megaw ${ }^{45}$ ). Using this model, along with temperature-dependent data from literature, we have plotted the expected fractional change in domain width from that at room temperature $\left(\Delta w / w_{0}\right)$ as a function of the temperature [expressed as a fraction of the Curie temperature $\left(T / T_{c}\right)$ ] in Fig. 3(b). In agreement with our observations (and those found in the literature), the domain width is almost constant across much of the temperature range; however, unlike most previous observations, the model predicts a significant increase in width close to $T_{c}$. This is clearly in qualitative agreement with our observations.

While Eq. (1) determines equilibrium values for domain width, the challenge faced by the system in reaching equilibrium is nicely illustrated by a sketch of the free energy as a function of domain width at two different temperatures just below $T_{c}$ (see Fig. 4). Here the form of the free energy function $(F)$ is taken to be that stated by Landau and Kittel, ${ }^{38,39}$ resulting from tension between a surface energy density term $[U(T)]$, which drives domain formation in the first place, and the energy cost associated with the creation of domain walls:

$$
F=U(T) w+\frac{\gamma(T) d}{w} .
$$

Under a thermal gradient that is not perfectly perpendicular to the domains, a single domain wall will simultaneously experience a range of temperatures along its length and will therefore be subjected to a variety of free energy landscapes (for example, close to the phase transition point, a wall will be subjected to both those free energy functions illustrated in Fig. 4 at the same time, but at different locations). A constant

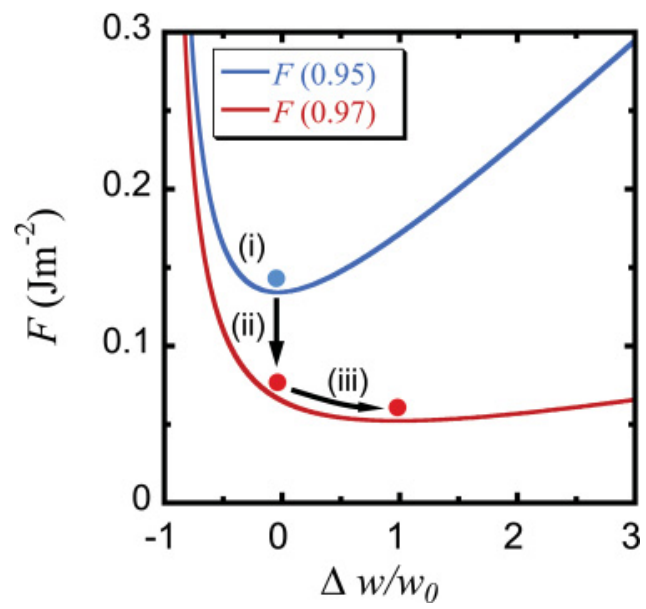

FIG. 4. (Color online) Modeled free energy curves as a function of the change in domain width. On heating, at $T / T_{c}=0.95$, the free energy function (blue curve) shows an equilibrium at (i). If the temperature is increased to $T / T_{c}=0.97$, then the free energy landscape changes (red curve) to give a much shallower "potential well" and an equilibrium domain width that is significantly increased. Under a general thermal gradient (which is nonperpendicular to the domains themselves), each domain wall will experience a range of energy functions at different locations along its length. Simultaneously satisfying equilibrium at all points along the domain wall requires domain annihilation. 
(a)
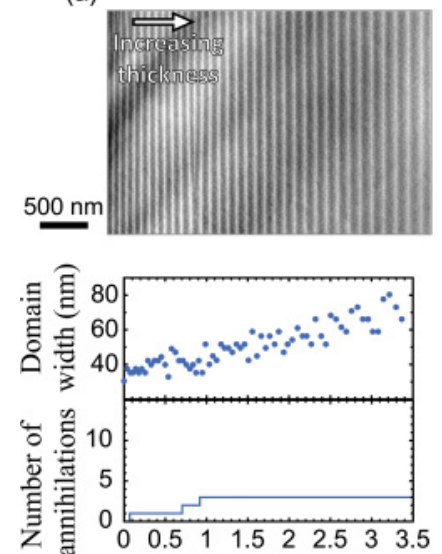

Position along gradient $(\mu \mathrm{m})$ (b)

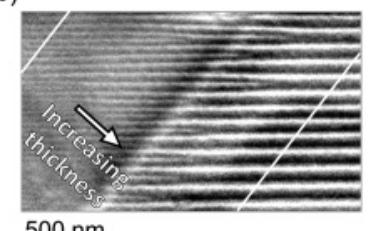
$500 \mathrm{~nm}$

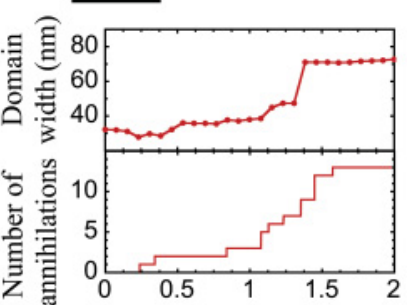

Position along gradient $(\mu \mathrm{m})$

FIG. 5. (Color online) Scanning transmission electron microscopy (STEM) images showing domain width changes with increasing thickness of the single crystal lamellae. In (a), the thickness gradient is perpendicular to the orientation of the domain walls and the width changes continuously with few annihilation events. Conversely, in (b), the thickness gradient is at a high angle to the domain wall orientation leading to many annihilation events and discontinuous domain width changes. Data displayed in the lower graphs are collected from between the two white lines on the STEM image.

number of domain walls will therefore not be able to satisfy the equilibrium periodicity conditions associated with all such landscapes simultaneously, without the creation or annihilation of domains. Once an annihilation event has taken place, the energy landscape changes as the equilibrium domain period is achieved. Only when the thermal gradient has no components parallel to the domain walls will the system be able to attain equilibrium domain periodicities at all locations without such domain creation or annihilation events.

We illustrate this in a directly analogous situation. Figure 5 shows domain patterns formed, after heating and cooling through the Curie temperature, in FIB-machined single-crystal thin films of $\mathrm{BaTiO}_{3}$ in which thickness gradients have been deliberately cut (the sample is locally wedge-shaped in cross-section at locations indicated in the figure). It can be clearly seen that changes in domain periodicity, induced by thickness changes, can be accommodated through both continuous variations in domain wall separation and through annihilation of domains. Crucially, it is the orientation of any thickness gradient with respect to the domain walls that determines whether the required change in domain period is accommodated through continuous change in domain wall separation or discrete annihilation events. In Fig. 5(a), where the thickness gradient is approximately perpendicular to the domain walls, few annihilation events occur and the domain width predominantly changes continuously. Conversely, in Fig. 5(b), the thickness gradient is strongly nonperpendicular

(approximately at an angle of $45^{\circ}$ ) to the domain walls and many annihilation events are observed.

The orientation of any temperature gradient with respect to the domain walls will therefore determine whether domain annihilation is the preferred mechanism for allowing periodicity changes. The quasistatic (inertia effects can be discounted as thermal equilibrium is reached in each temperature step) temperature gradient imposed on this sample imaged by AFM has been shown to generate the same domain annihilation behavior as that observed under thickness gradients. Although, it should be noted that the domain width is a linear function of the square root of thickness, whereas it is a complex function of temperature [see Fig. 3(b)]. We also note similarities between the work presented here, namely, the behavior of periodic structure close to a phase transition and that of staging transformations in intercalation compounds. ${ }^{46}$ In both cases, discontinuous behavior of periodic features are expected.

It is suggested that domain annihilation under thermodynamic gradients must be a universal system response; gradients in stress and electric field are obvious alternatives to those in size and temperature. This realization may be increasingly important as the use of gradient-induced functional behavior, such as flexoelectricity, ${ }^{4-50}$ becomes more widespread and technologically relevant.

\section{SUMMARY AND CONCLUSIONS}

In summary, $90^{\circ}$ a-c domains in single-crystal $\mathrm{BaTiO}_{3}$ have been investigated close to the ferroelectric-to-paraelectric phase transition using AFM. Changes in domain period within this region have been explained by adapting established models for ferroelastics to include temperature-dependent quantities. The equilibrium domain period is shown to be a critical function of temperature and this gives rise to domain annihilation events when thermal gradients exist. Comparison between the observations made under this thermal gradient and those made in thin $\mathrm{BaTiO}_{3}$ platelets where a thickness gradient exists, demonstrate that changing domain period through domain annihilation is a general feature when gradient vectors are not perpendicular to the domain walls with which they interact.

\section{ACKNOWLEDGMENTS}

Many thanks to L. N. McCartney at NPL for useful discussions. J.M.G. and L.Mc.G. gratefully acknowledge the Department for Employment and Learning (DEL) in N. Ireland for studentship support. J.M.G. acknowledges grant support from the Engineering and Physical Sciences Research Council (EPSRC) (Grant EP/F004869/1). T.L.B. and M.G.C. gratefully acknowledge funding from the National Measurement Office, Innovation Research and Development (IRD) program Nanoferroelectrics (113663). A.S. acknowledges financial support from EPSRC (Grant EP/H00307X/1).
*Current address: Ceramics Laboratory, EPFL-Swiss Federal Institute of Technology, Lausanne 1015, Switzerland.

${ }^{1}$ J. F. Scott, Ferroelectric Memories (Springer-Verlag, Berlin 2000).
${ }^{2}$ D. Damjanovic and M. Demartin, J. Phys. Condens. Matter 9, 4943 (1997).

${ }^{3}$ R. Runge, Y. Nozaki, Y. Otani, H. Miyajima, B. Pannetier, T. Matsuda, and A. Tonomura, J. Appl. Phys. 79, 5075 (1996). 
${ }^{4}$ P.-O. Jubert, J.-C. Toussaint, O. Fruchart, C. Meyer, and Y. Samson, Europhys. Lett. 63, 132 (2003).

${ }^{5}$ J. Raabe, C. Quitmann, C. H. Back, F. Nolting, S. Johnson, and C. Buehler, Phys. Rev. Lett. 94, 217204 (2005).

${ }^{6}$ A. Masseboeuf, O. Fruchart, J. C. Toussaint, E. Kritsikis, L. BudaPrejbeanu, F. Cheynis, P. Bayle-Guillemaud, and A. Marty, Phys. Rev. Lett. 104, 127204 (2010).

${ }^{7}$ N. Balke, S. Choudhury, S. Jesse, M. Huijben, Y. H. Chu, A. P. Baddorf, L. Q. Chen, R. Ramesh, and S. V. Kalinin, Nat. Nanotechnology 4, 868 (2009).

${ }^{8}$ L. J. McGilly, A. Schilling, and J. M. Gregg, Nano Lett. 10, 4200 (2010).

${ }^{9}$ Y. Ivry, D. P. Chu, J. F. Scott, and C. Durkan, Phys. Rev. Lett. 104, 207602 (2010).

${ }^{10}$ R. K. Vasudevan, Y.-C. Chen, H.-H Tai, N. Balke, P. Wu, S. Bhattacharya, L. Q. Chen, Y.-H. Chu, I.-N. Lin, S. V. Kalinin, and V. Nagarajan, ACS Nano 5, 879 (2011).

${ }^{11}$ C. T. Nelson, B. Winchester, Y. Zhang, S.-J. Kim, A. Melville, C. Adamo, C. M. Folkman, S.-H. Baek, C.-B. Eom, D. G. Schlom, L.-Q. Chen, and X. Pan, Nano Lett. 11, 828 (2011).

${ }^{12}$ C.-L. Jia, K. W. Urban, M. Alexe, D. Hesse, and I. Vrejoiu, Science 331, 1420 (2011).

${ }^{13}$ N. Balke, B. Winchester, W. Ren, Y. H. Chu, A. N. Morozovska, E. A. Eliseev, M. Huijben, R. K. Vasudevan, P. Maksymovych, J. Britson, S. Jesse, I. Kornev, R. Ramesh, L. Bellaiche, L. Q. Chen, and S. V. Kalinin, Nat. Phys. 8, 81 (2012).

${ }^{14}$ R. G. P. McQuaid, L. J. McGilly, P. Sharma, A. Gruverman, and J. M. Gregg, Nat. Commun. 2, 404, (2011).

${ }^{15}$ H. Fu and L. Bellaiche, Phys. Rev. Lett. 91, 257601 (2003).

${ }^{16}$ I. I. Naumov, L. Bellaiche, and H. Fu, Nature (London) 432, 737 (2004).

${ }^{17}$ W. J. Merz, Phys. Rev. 76, 1221 (1949).

${ }^{18}$ P. M. Weaver, M. G. Cain, and M. Stewart, Appl. Phys. Lett. 96, 142905 (2010).

${ }^{19}$ P. M. Weaver, M. G. Cain, and M. Stewart, J. Phys. D 43, 165404 (2010).

${ }^{20}$ F. De Guerville, I. Luk'yanchuka, L. Lahoche, and M. El Marssia, Mat. Sci. Eng. B 120, 16 (2005).

${ }^{21}$ I. A. Luk'yanchuk, L. Lahoche, and A. Sené, Phys. Rev. Lett. 102, 147601 (2009).

${ }^{22}$ M. L. Mulvihill, K. Uchino, Z. Li, and W. Cao, Phil. Mag. B 74, 25 (1996).

${ }^{23}$ S. V. Kalinin and D. A. Bonnell, J. Appl. Phys. 87, 3950 (2000).
${ }^{24}$ S. V. Kalinin and D. A. Bonnell, Appl. Phys. Lett. 78, 1116 (2001).

${ }^{25}$ E. Z. Luo, Z. Xie, J. B. Xu, I. H. Wilson, and L. H. Zhao, Phys. Rev. B 61, 203 (2000).

${ }^{26}$ P. Lehnen, W. Kleemann, Th. Woike, and R. Pankrath, Phys. Rev. B 64, 224109 (2001).

${ }^{27}$ H. Okino, J. Sakamoto, and T. Yamamoto, Jpn. J. Appl. Phys. 42, 6209 (2003).

${ }^{28}$ V. V. Shvartsman and A. L. Kholkin, Phys Rev B 69, 014102 (2004).

${ }^{29}$ X. Y. Liu, Y. M. Liu, S. Takekawa, K. Kitamura, F. S. Ohuchi, and J. Y. Li, J. Appl. Phys. 106, 124106 (2009).

${ }^{30}$ P. W. Forsbergh Jr., Phys. Rev. 76, 1187 (1949).

${ }^{31}$ T. J. Parker and J. C. Burfoot, Br. J. Appl. Phys. (J. Phys. D) 2, 1168 (1969).

${ }^{32}$ M. Varioglu, E. Ustundag, N. Tamura, and J. L. Jones, J. Appl. Phys. 107, 064101 (2010).

${ }^{33}$ M. Foeth, P. Stadelmann, and M. Robert, Physica A 373, 439 (2007).

${ }^{34}$ A. Schilling, T. B. Adams, R. M. Bowman, J. M. Gregg, G. Catalan, and J. F. Scott, Phys. Rev. B 74, 024115 (2006).

${ }^{35}$ G. Arlt and P. Sasko, J. Appl. Phys. 51, 4956 (1980).

${ }^{36}$ E. K. H. Salje and Y. Ishibashi, J. Phys. Condens. Matter 8, 8477 (1996).

${ }^{37}$ M. Takashige, S. Hamazaki, Y. Takahashi and F. Shimizu, Jpn. J. Appl. Phys. 38, 5686 (1999).

${ }^{38}$ L. Landau and E. Lifshitz, Phys. Z. Sowjetunion 8, 153 (1935).

${ }^{39}$ C. Kittel, Phys. Rev. 70, 965 (1946).

${ }^{40}$ L. Roitburd, Phys. Status Solidi A 37, 329 (1976).

${ }^{41}$ N. A. Pertsev and A. G. Zembilgotov, J. Appl. Phys. 78, 6170 (1995).

${ }^{42}$ W. Pompe, X. Gong, Z. Suo, and J. S. Speck, J. Appl. Phys. 74, 6012 (1993).

${ }^{43}$ P. Marton, I. Rychetsky, and J. Hlinka, Phys. Rev. B 81, 144125 (2010).

${ }^{44}$ L. Dong, D. S. Stone, and R. S. Lakes, Phil. Mag. 90, 23 (2010).

${ }^{45}$ H. D. Megaw, Proc. Royal Soc. A. 189, 261 (1947).

${ }^{46}$ P. Bak and G. Forgacs, Phys. Rev. B 32, 7535 (1985).

${ }^{47}$ A. K. Tagantsev, Phys. Rev. B 34, 5883 (1986).

${ }^{48}$ G. Catalan, L. J. Sinnamon, and J. M. Gregg, J. Phys. Condens. Matter 16, 2253 (2004).

${ }^{49}$ W. H. Ma and L. E. Cross, Appl. Phys. Lett. 81, 3440 (2002).

${ }^{50}$ G. Catalan, A. Lubk, A. H. G. Vlooswijk, E. Snoeck, C. Magen, A. Janssens, G. Rispens, G. Rijnders, D. H. A. Blank, and B. Noheda, Nat. Mater. 10, 963 (2011). 